\title{
COMPACT H-SHAPE MICROSTRIP PATCH ANTENNA USING HFSS
}

\author{
Miss. A. A. Bellale \\ Department of Digital Communication \\ M.B.E.S. COE, Ambajogai, Maharashtra, India.
}

\author{
Prof. V. V. Yerigeri, Prof. S. M. Patil \\ Department of Post Graduation \\ M.B.E.S. COE, Ambajogai, Maharashtra, India.
}

\begin{abstract}
As the recent technology emerges in wireless communication increase in antenna research also found in the recent decades. The demand is for the high quality antennas having compact structure with superior gain and also have multiple bands. There is also increase in online services found by service providers as all business facilities are now available online. These services are continuously striving for better connectivity with high bandwidth. And so for technologies becoming cheaper as competition increases. This paper proposes, $\mathrm{H}$-shaped patch antenna fabricated with using FR4 substrate and demonstrated using simulations. Also it's tested for various wireless applications like WiMAX, broadcasting, etc. Developed antenna deals with compactness, design complexity and reduces the thickness, the mass, and makes it cheaper as compare to other transmitarray antennas.
\end{abstract}

Keywords - transmitarray, unit cell element, antenna beamsteering

\section{INTRODUCTION}

Now a days the transmitarray antenna available in various shapes as per the requirement of the industrial applications. The various shapes are derived on the basis of their transmission signals direction. Each antenna of a specific shape has their own transmission direction pattern, performance measurement, gain properties and their specific applications. Its worth to say that transmitarray antennas performing a vital role to serve the recent advances in wireless communication technology. The advances and developments can be seen for microstrip type antenna in ending of 70's and found to be great achievement in the starting of 80 's by establishing various design structures, patterns and models according to antenna unit cells and array patterns. In the recent decades the printed antennas are researched a lot due to their large advantages like lighter weight, compactness, cheaper, ease of installation and high performance upon radiating systems [1-4].The design of microstrip patch antenna includes a structured patch for radiation at substrateand ground plane for support as per general antenna desgn. Patching is performed on metal used in fabrication in general. The radiating patch and the feed lines are usually photo etched on the dielectric substrate Microstrip patch antennas radiate primarily because of the fringing fields between the patch edge and the ground plane[18].Due to this, it's become noncontacting in nature. Contacting method includes radio frequency power to be served directly patch radiation by integrating microstrip line or probe feed [5-9]. At another side in the non-contacting method electromagnetic field is paired to hand over power among the microstrip line and radiating patch this includes proximity feeding and aperture feeding. Microstrip antennas are considered by a larger number of physical parameters than conventional microwave antennas. These antennas can be fabricated according to radiation requirement to meet geometrical shapes and dimensions but rectangular and circular resonant patches are casted extensively in many applications. Communication is a vital part of business growth, personal growth, and overall growth where there is direct in person contact matters [10]. Infoexchange can be mostly done with electronic media at everywhere by the means of radio, television and mobile phones broadly categorized as telecommunication systems. These systems are driven by the means of receiver and transmitter concepts in which transmitter communicates to the outside world by sending signals and signals consumed by receiver from the outside world. Sometimes transmitter and receiver are combined together to form a transceiver which plays both role of the system. The communication signals are the radio frequency energy.

The remaining paper is organized as follows. Related work and broad studies are given in section II. Section III describes proposed work and also states microstrip line feed. Design and analysis according to the result requirements are given in section IV with gain and other result parameters. Conclusions are given in section $\mathrm{V}$.

\section{RELATED WORK}

Parametric study of the planar monopole antenna is necessary to understand the performances under various aspects of designs and their respected observations. Due to the various features like light weight, low volume, thin size the microstrip monopole antenna has become the first choice of the manufacturer possessing very large bandwidth capability whereas a simple microstrip antenna suffers from narrow impedance bandwidth and low power-handling capability like limitations. An antenna design with four layers working at the frequency of $30 \mathrm{GHz}$ is proposed. The unit cell element of the array is consisted of dual-resonant made up of double square 
ring. The phase variation is increased for the each layer by using a double ring of two resonances and also the magnitude of transmission is preserved. The process of single layered transmitarray element and the poured connection of four layer is defined which hints to $50 \%$ increase in the $-1 \mathrm{~dB}$ gain bandwidth upon early antennas[11-15].Even though there is number of compensations of planar transmitarray antennas as compared to conventional systems, these possesses narrowercapacity of bandwidth. In this work we have concentrated on the improvement of capacity bandwidthof transmitarray antennas. This is done by controlling phase of transmission range and the optimization of phase of distribution on the transmitarray aperture. For the reconsideration of the developed design two quad-layer transmit arrayscoupled with double square loop elements are manufactured. These are further fabricated, and tested at $\mathrm{Ku}$ band. Phase distribution of the transmission is improved for these antennas. These are varied only in the range of transmission. We have examined dual linearly polarized transmitarray antennas having wideband output in X-band. These took us a long time to manufacture it and analyses it according to the parameters available with and also the parameters tested with. The designs and structures are constructed on unit cell elements considering bandpass response whereas the design also includes three conducting layers. These layers are printed on two similar dielectric substrates attached with a bonding film. The results of experiment validated outstanding polarization independence, with as per the expectation of performance. These performances appropriately conserved with radiation characteristics for every polarization. The work represents wideband transmitarray antenna constructed as a double-layer through two degrees of freedom. Four metal viaswithtwo patches for with two degrees of freedom (TDF) are observed to get the better results as compared to conventional antennas [16-17]. The phase shift of range 360-degree is appreciated with dual degrees of freedom. Higher performance with superior characteristics is measured in proposed work with fabrication and design to examine the output gain and radiation pattern as we have found to overcome in the previous transmitarray antennas. The elements are selected in the manufacturing are considered to be giving the proper results as we have constructed on dielectric substrate with relative permittivity $\varepsilon r$ and loss tangent $\delta$. Structure design with actual measurements of the element are taken in $L \times L \times H$ units. Wedescribehere the length of LC as the first degree of freedom (FDF) and the second degree of freedom (SDF) as the length of LS. As the LC fluctuates from $4 \mathrm{~mm}$ to $8 \mathrm{~mm}$ and LS fluctuates from $0.6 \mathrm{~mm}$ to $4 \mathrm{~mm}$, the FDF and SDF cases can correct the 300-degree and 130-degree phase shift range, correspondingly. The FDF accomplishes the start degree of phase shift range, the SDF concludes the last degree of phase shift range. To execute phase compensation of 300 degree we must use the cross-dipole as the structure element so that the fabrication can be simplified whereas for phase compensation between 300 degrees and 360 degrees we need to use spiraldipole in the element design. Through coalescing these two parameters, we can acquire a 360-degree phase shift range. Four metal vias passing through the entire dielectric substrate are used to enrich the coupling strength (fig. 1) [1-3].

Metal vias have a great importance towards the transmission magnitude and TDF makes the element achieve 360-degree phase shift. Therefore, the novel TDF element is used to construct a double-layer wideband transmitarray antenna. The transmitarray antenna has $1-\mathrm{dB}$ gain bandwidth of $15.5 \%$ and peak efficiency of $55 \%$. The progress of the transmitarray antennas is depends upon multi-layer structure, which can realize stable transmission magnitude and provide 360-degree phase shift in the whole band. Multi-layer structure increases the processing difficulty and cost. [19]

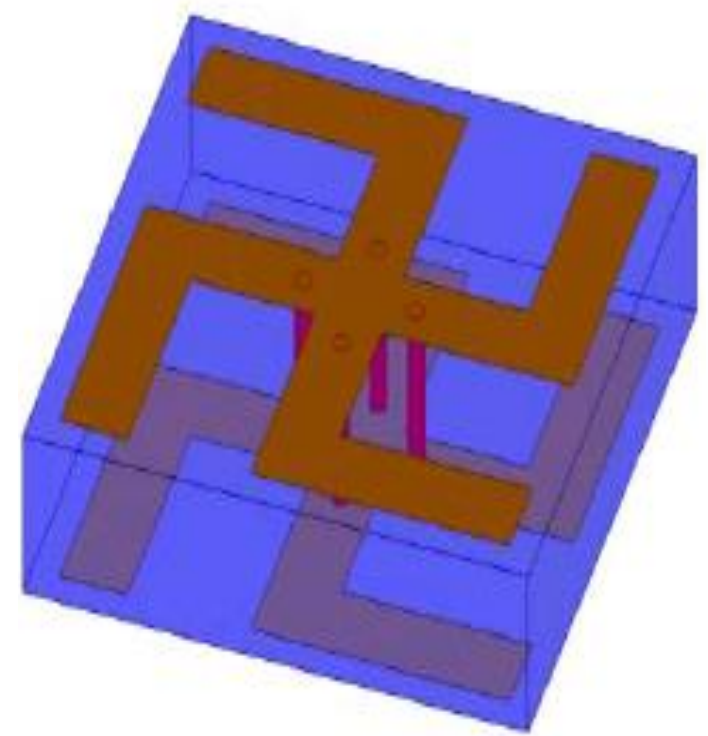

Fig.1: Double-Layer Wideband Transmitarray Antenna

To solve such problems we have to follow an novel approach of the double-layer structure which also makes easier. The double layered design we have to consider the transmission magnitude and phase shift first.

\section{PROPOSED SYSTEM}

In this method, a compact size patch antenna is proposed with dielectric substrate as FR-4 Epoxy and dimensions are based on resonant frequency. The partial ground plane intensely combined through the radiating component. In addition the aimed antenna is proficient at associating numerous resonances prominent to the widespread functioning band. To meet the better performances and higher bandwidth with higher gain the antenna must be modified accordingly by considering its applications like Wi-fi, WiMAX, digital broadcasting, radar and navigation services etc. Fine and better design structures always makes reliable system 
requirements and advances the overall system performance by reducing the overall load on the system. FR4-Epoxy is used as a substrate for low frequency design. In order to further reduce the size of implantable antennas, used much higher permittivity substrate to design implantable antennas.FR4 (or FR4) is a NEMA grade designation for glass-reinforced epoxy laminate material. FR-4 is a compound material consst of woven fiberglass cloth with an epoxy resin binder that is fire resistant (self-extinguishing). The microwave and RF features of FR4 substrates. FR4 epoxy glass substrates are the material of choice for most PCB applications. These materials are cheaper and possesses strengthen properties makes it easy to fabricate with various electronic component appliances. This is a grade designation assigned to glass reinforced epoxy laminate sheets, tubes, rods and printed circuit boards (PCB). With dielectric constant equal to $4.4, \mathrm{FR} 4$ is a composite material composed of woven fiberglass cloth with an epoxy resin binder that is flame resistant (self-extinguishing). FR4 glass epoxy is a popular and versatile high pressure thermoset plastic laminate grade with good strength to weight ratios. With near zero water absorption, FR4 is most commonly used as an electrical insulator possessing considerable mechanical strength. A very important aspect of an antenna is the height of the substrate. This has to be kept at the minimum as our antenna has to be used in cell phones. This is a key component affecting the spatial geometry, which is based on the ratio $\mathrm{w} / \mathrm{h}$ where $h$ is the height of the substrate and $w$ is its width.



Fig.2: Proposed Antenna Structure

For a proper comparison of return loss and gain, the height of all substrates has been taken as $1.5 \mathrm{~mm}$. Shorter wavelength can be observed with greater operating frequency and required smaller designs in volume. For greater data-rate communication a high capacity bandwidth with greater operating frequency is required. (fig. 2)

\section{A) Microstrip Line Feed}

A conducting strip can be joined straight to the edge of microstrip patch in such a feed technique. Strip which is conducting is kept smaller in design of width by comparing to the patch. This leads to the advances of feed etching on the same substrate. This gives us a planar structure for the antenna design. To match the impedance of the feed line to the patch there is inset cut is maintained, this all is done without the need for any extra element. The inset position is systematically controlled to achieve this performance. (fig. 3)

\section{DESIGN AND RESUlts}

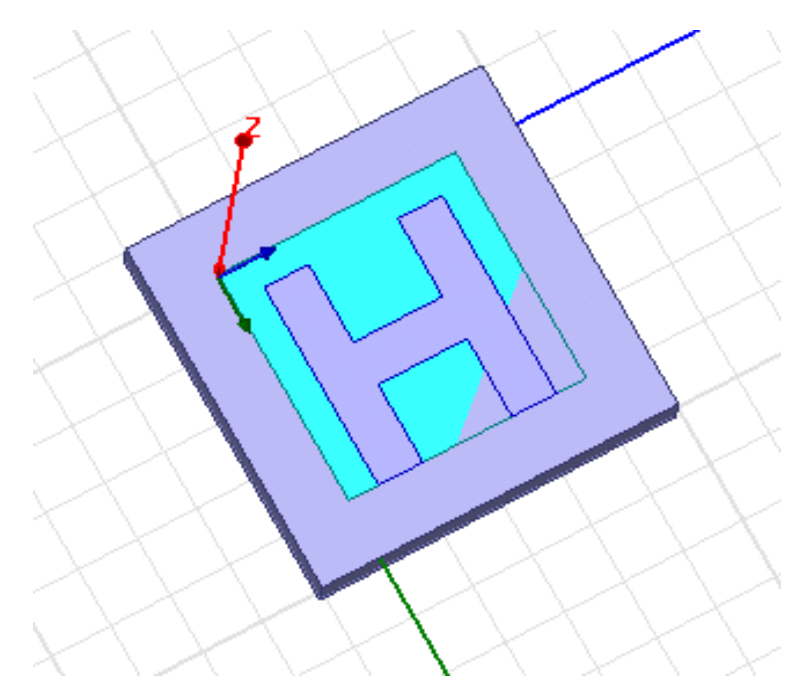

Fig.3: H-Design

\section{B) Voltage Standing Wave Ratio (VSWR):}

Voltage Standing Wave Ratio (VSWR) is an signal of the superiority of the impedance contest. VSWR which is also known as SWR. A high VSWR is represents the signal is reflected prior to being radiated by the antenna. VSWR and reflected power are different ways of measuring and expressing the same thing. (fig. 4-5) 




Fig.4: VSWR1

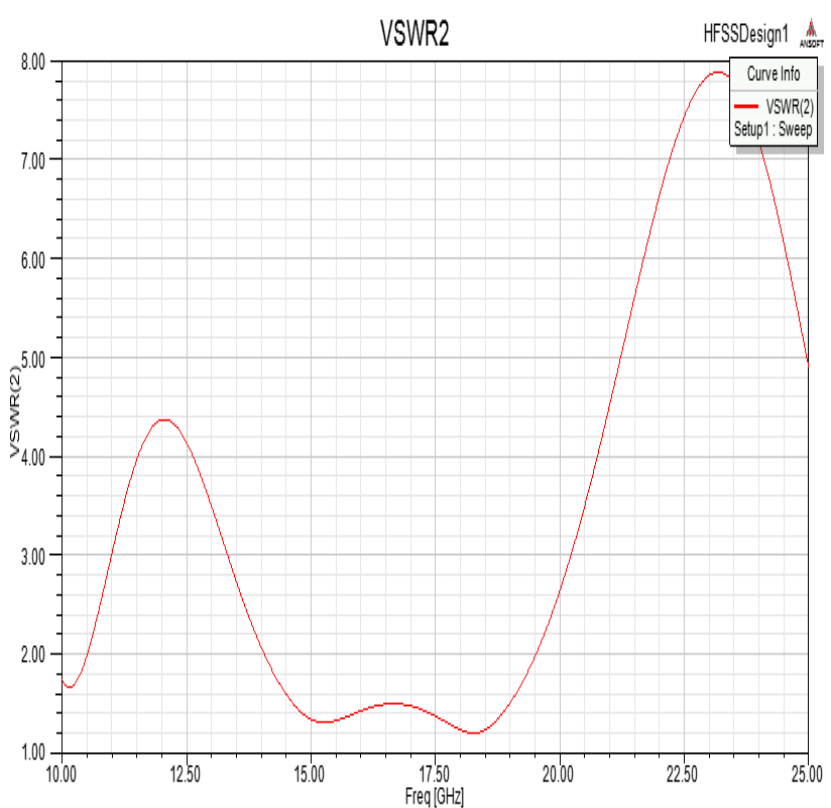

Fig. 5: VSWR2

\section{C) Gain:}

Gain is an antenna property deals with an antenna's ability to direct its radiated power in a desired direction, or synonymously, to receive energy preferentially from a preferred direction. The gain of the proposed antenna is 11.4 dB. (fig. 6)

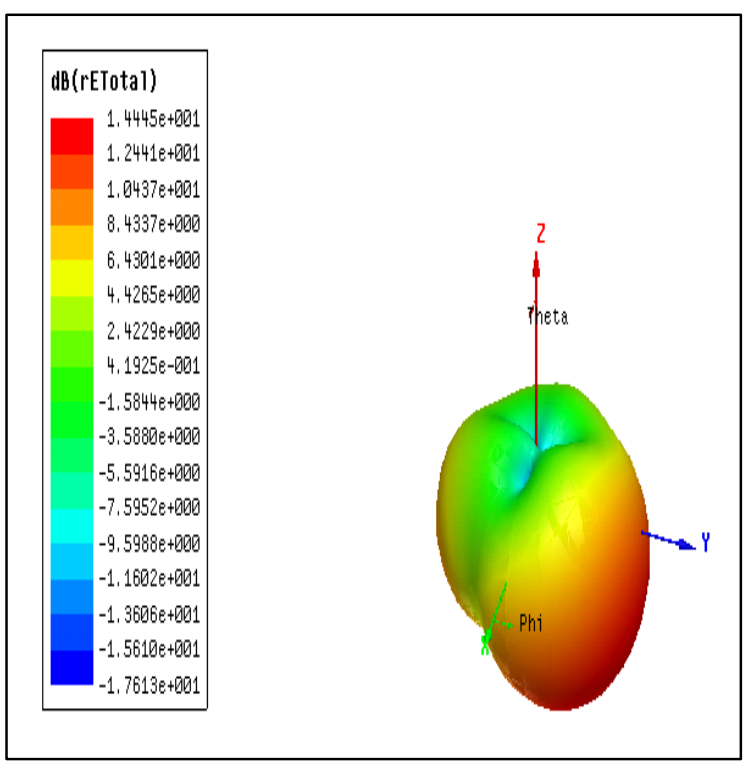

Fig.6: Gain

\section{D) Radiation Pattern:}

For a linearly polarized antenna, performance is often described in terms of its Principal E- and H-plane patterns. The E-plane is defined as "the plane containing the electricfield vector and the direction of the maximum radiation." The H-plane as "the plane containing the magnetic field vector and the direction of the maximum radiation."The radiation pattern of an antenna is a plot of the far-field radiation properties of an antenna as a function of the spatial co-ordinates which are specified by the elevation angle $(\theta)$ and the azimuth angle $(\varphi)$. (fig. 7)

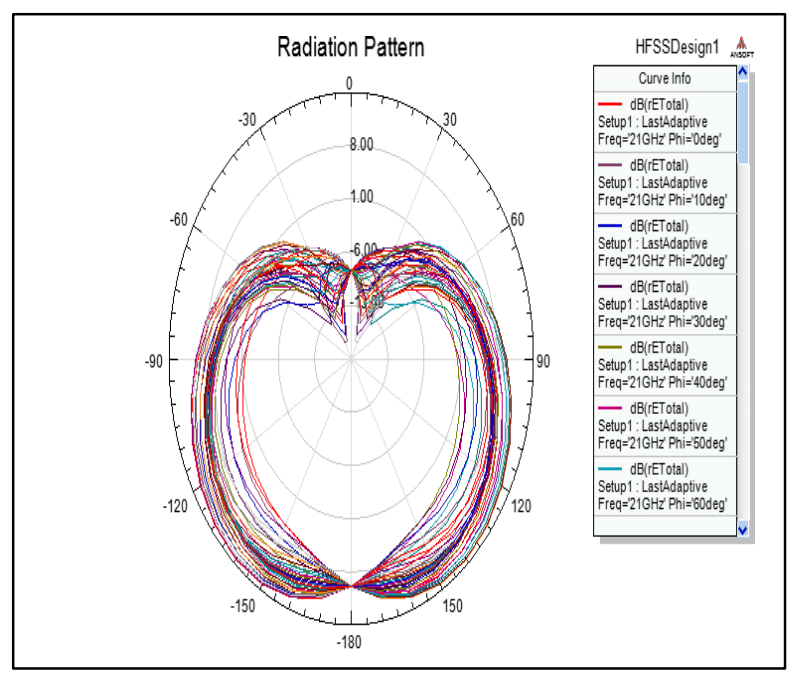

Fig.7: Radiation Pattern 


\section{International Journal of Engineering Applied Sciences and Technology, 2020 Vol. 5, Issue 3, ISSN No. 2455-2143, Pages 637-642 \\ Published Online July 2020 in IJEAST (http://www.ijeast.com)}

\section{E) Return Loss:}

It is a parameter which indicates the amount of power that is "lost" to the load and does not return as a reflection. Hence a parameter to indicate how well the matching between the transmitter and antenna has taken place. The bandwidth can be calculated from the return loss (RL) plot. (fig. 8-9)

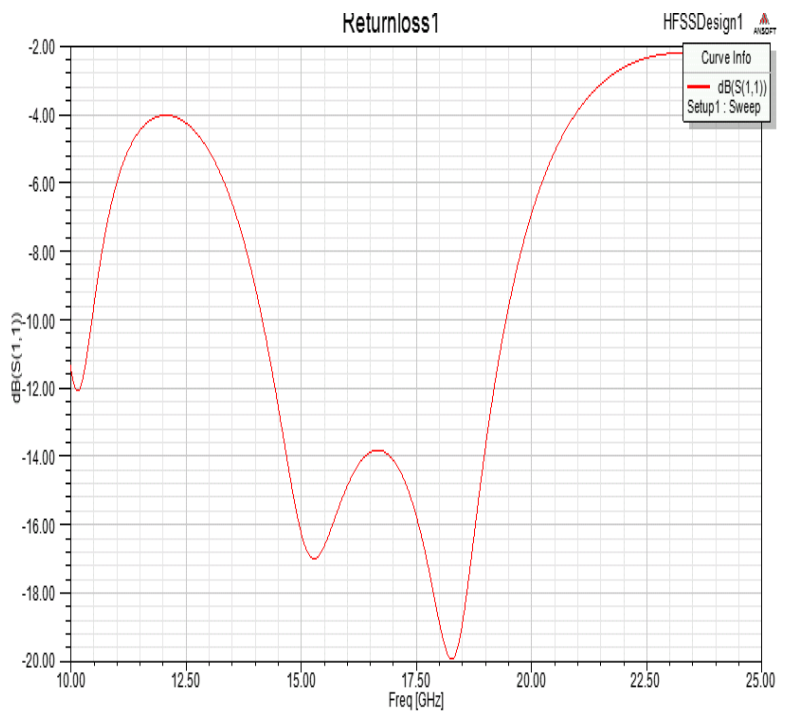

Fig.8: Return Loss1

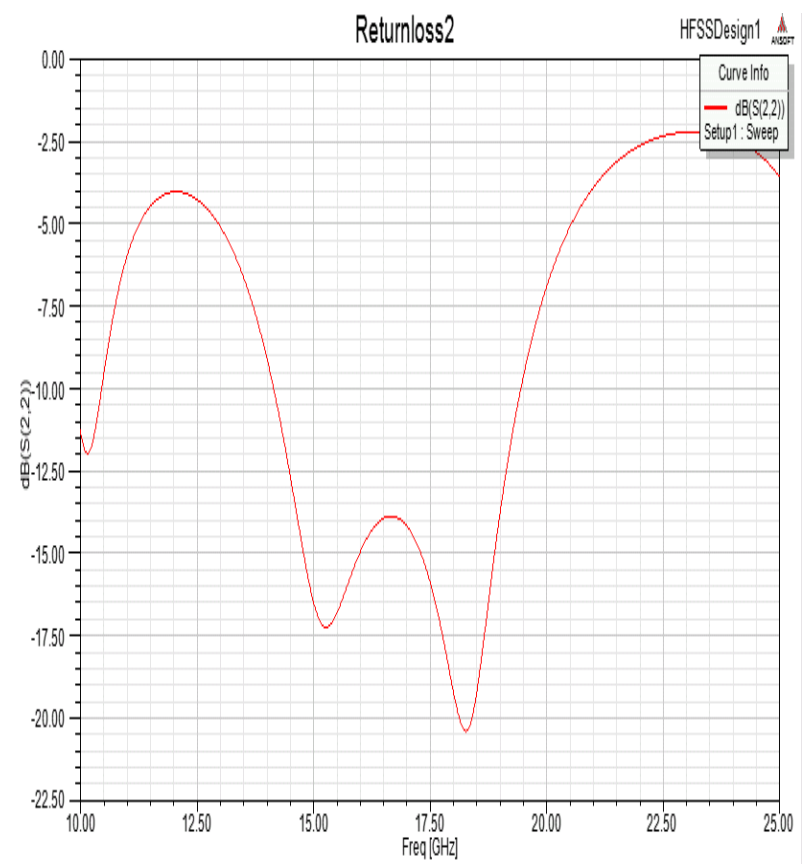

Fig. 9: Return Loss2

\section{CONCLUSION}

This project proposes the design of H-shaped patch antenna is designed. The antenna has been evaluated using HFSS software very high gain and excellent voltage standing wave ratio. The optimization strategy employed for investigating the antenna characteristics shows high efficiency to achieve a high performance and high accuracy.

\section{REFERENCES}

[1] C. G. M. Ryan et al., (2010)“A wideband transmitarray using dual-resonant double quare rings," IEEE Trans. Antennas Propag., vol. 58, no. 5, pp. 1486-1493.

[2] A. H. Abdelrahman, P. Nayeri, A. Z. Elsherbeni, and F. Yang,(2015)"Bandwidth improvement methods of transmitarray antennas," IEEE Trans. Antennas Propag., vol. 63, no. 7, pp. 2946-2954.

[3] B. Rahmati, H. R. hassani, (2015) "high-efficient wideband slot transmitarray antenna," IEEE Trans. Antennas Propag., vol. 63, no. 11, pp. 5149-5155.

[4] K. Pham, N. T. Nguyen, (2016) "design of wideband dual linearly polarized transmitarray," IEEE Trans. Antennas Propag., vol. 64, no. 5, pp. 2022-2026.

[5] C. Jouanlanne, A. Clemente, (2017) "wideband linearly polarized transmitarray antenna for $60 \mathrm{GHz}$ backhauling," IEEE Trans. Antennas Propag., vol. 65, no.3, pp. 1440-1445.

[6] A. H. Abdelrahman, A. Z. Elsherbeni, and F. Yang, (2014) "High-gain and broadband transmitarray antenna using triplelayer spiral dipole elements," IEEE Antennas Wireless Propag. Lett., vol. 13, pp. 1288-1291.

[7] Guang Liu, Hong-jian Wang, J.S. Jiang, (2015) "A highefficiency transmitarray antenna using double split ring slot elements," IEEE Antennas Wireless Propag. Lett., vol. 14, pp. 1415-1418.

[8] B. Rahmati, H. R. Hassani,(2015) "high-efficient wideband slot transmitarray antenna," IEEE Trans. Antennas Propag., vol. 63, no. 11, pp. 5149-5155.

[9] B. Rahmati, H. R. Hassani, (2015) "low-profile slot transmitarray antenna," IEEE Trans. Antennas Propag., vol.63,no.1,pp. 178-180.

[10] J. G. Nicholls, S. V. Hum, (2016) "full-space electronic beam-steering transmitarray with integrated leaky-wave feed," IEEE Trans. Antennas Propag.,vol.64,no.4,pp. 1473-1481.

[11] H. Nematollahi, J-J Laurin, (2015) "design of broadband transmitarray unit cells with comparative study of different numbers of layers," IEEE Trans. Antennas Propag.,vol.63,no.8,pp. 3410-342.

[12] Chao Tian, Y-C Jiao, Guang Zhao, (2017) “circularly polarized transmitarray antenna using low-profile dual-linearly polarized elements," IEEE Antennas Wireless Propag. Lett., vol. 16, pp. 465-468.

[13] J. Y. Lau, S. V. Hum, (2011) "analysis and characterization of a multipole reconfigurable transmitarray 
element," IEEE Trans. Antennas Propag.,vol.59,no.1,pp. 7079.

[14] E. G. Plaza, G. L, S. Loredo, (2017) “an ultrathin 2-bit near-field transmitarray lens," IEEE Antennas Wireless Propag. Lett., vol. 16, pp. 1784-1787.

[15] Wenxing An, ShenhengXu, (2016) "a double-layer transmitarray antenna using malta crosses with vias," IEEE Trans. Antennas Propag.,vol.64,no.3,pp. 1120-1125.

[16] S. H. Zainud-Deen, S. M. Gaber, H. A. Malhat, and K. H. Awadalla, "Multilayer dielectric resonator antenna transmitarray for near-field and far-field fixed RFID reader," in Proc. 29th Nat. Radio Sci. Conf., 2012, pp. 81-88.

[17] W. Imbriale, S. Gao, and L. Boccia, Eds., (2012) Space Antenna Handbook. New York NY, USA: Wiley.

[18] S.Dwivedi, A.Rawat, R. N. Yadav.(2013) "Design of Ushape microstrip patch antenna forWiMAX applications at 2.5 GHz", 2013 Tenth International Conference on Wireless and Optical Communications Networks (WOCN).

[19] Xiangjie Yi, Tao Su, Xi Li, Bian Wu, Lin Yang. (2019)

"A Double-Layer Wideband Transmitarray Antenna Using Two Degrees of Freedom Elements Around 20 GHz" , IEEE Transactions on Antennas and Propagation. 